\title{
SELF-REFERENCE BEAMFORMING FOR DS-CDMA COMMUNICATION SYSTEMS
}

\author{
Daniel Pérez Palomar and Miguel Angel Lagunas \\ Department of Signal Theory and Communications \\ Universitat Politècnica de Catalunya \\ c/ Jordi Girona, 1-3, Mòdul D5, Campus Nord UPC \\ 08034 Barcelona (Spain) \\ e-mail: daniel@gps.tsc.upc.es
}

\begin{abstract}
In this paper, a self-reference beamforming algorithm, based on the inherent temporal redundancy structure presented by the spreading codes of Direct Sequence Code Division Multiple Access (DSCDMA) systems, is proposed. It is derived from a novel perspective of chip level cross-correlation properties.

The algorithm is shown to give the optimal solution in the sense of maximum Signal to Interference plus Noise Ratio (SINR). Block and adaptive approaches to solve the problem are given.

The method is tested against the well-known Temporal Reference Beamformer (TRB) combined with a decision-directed approach, showing a performance similar to it, despite no side information is being used.
\end{abstract}

\section{INTRODUCTION}

Code Division Multiple Access (CDMA) has been proposed as an efficient multiple access method for cellular and personal communication systems, e.g. IS-95 [1] and IS-665 [2]. It has been shown to offer higher capacity than the existing FDMA or TDMA methods but, in contrast to them, CDMA systems are interference limited.

Many different approaches have been devised to combat the Multi-Access Interference (MAI), which is originated by the rest of the users [3]. The MAl problem can be further intensified by the near-far effect, which is due to the different geographical situation (distance to the base station and multipath propagation) of the mobile users.

The conventional detector is simply a bank of matched filters. It assumes the MAI as additive white Gaussian noise (AWGN) and it is a decentralized detector (it does not use the information of all the users jointly). The optimum centralized multiuser detector, maximum likelihood detector, was proposed in [4]; but even for a small number of users, its computational cost is unacceptable. Thus, suboptimum but still multiuser-based methods have been proposed. One family of these suboptimum algorithms includes the so-called linear detectors (they perform a linear combination of the output of the matched filters to decouple the user's signals), such as the decorrelating detector $[5,6]$, the minimum meansquare error (MMSE) detector [7] and the polynomial expansion

This work was partially supported by the European Comission under Project IST-1999-11729 METRA; the Spanish Government (CYCIT) TIC98-0412, TIC98-0703; and the Catalan Government (CIRIT) 1998SGR $00081,1999 \mathrm{Fl} 00588$.
(PE) detector [8]. Another important family includes the subtractive interference cancellation detectors and comprises serial and parallel versions: successive interference cancelation (SIC) and parallel interference cancellation (PIC) [9]. They basically subtract a previous estimation of the user signals on an iterative basis, so that for each new estimation, each user sees a reduced amount of MAI.

In principle, all of the aforementioned methods were devised to combat the MAI based on a monosensor detection. Nevertheless, an approach to further increase the system capacity is the use of spatial processing with base station antenna arrays, also called Spatial Diversity Multiple Access (SDMA). By using spatial diversity at cell sites, it is possible to use adaptive receive/transmit beamformers for each user so that interference from/to other users is nulled out. In this way, after the beamforming stage, the previously named detectors will see a nearly interference-free environment due to the reduction of co-channel interference from within the own cell and neighboring cells.

Many efforts have been spent in this direction of multisensor detection approach during the last years, such as: the Temporal Reference Beamforming (TRB) [10] (which implies a loss of spectral and channel bandwidth efficiency due to the transmission of the reference signal), possibly combined with a decision-directed approach, which regenerates the reference signal at the receiver [11]. More sophisticated methods have been devised without the need of side information, such as [12], where the steering vector of the desired user is estimated using a subtraction of two matrices in which only the desired component survives; [13], in which a phased array is used after the MAI has been removed by means of a decorrelating detector; or the DEDYSS (DEcoupling of DYads of Spatial Signatures) algorithm [14], which estimates the steering vector of the users jointly.

In this paper, a blind beamforming algorithm suitable for DSCDMA systems is described. It relies on the fact that the pseudonoise (PN) sequence or spreading code can be used as an implicit temporal reference.

\section{TIME REFERENCE BEAMFORMER}

The Time Reference Beamformer (TRB) is a classical beamforming technique that makes use of a temporal reference, $d(t)$. This training signal allows the definition of an error between the output signal and the desired one $e(t)=d(t)-\mathbf{w}^{H} \mathbf{x}(t)$, where $\mathbf{w}$ is the beamvector and $\mathbf{x}(t)$ is the snapshot assuming narrowband and plane-wave signals. The Wiener solution of the beamformer, 


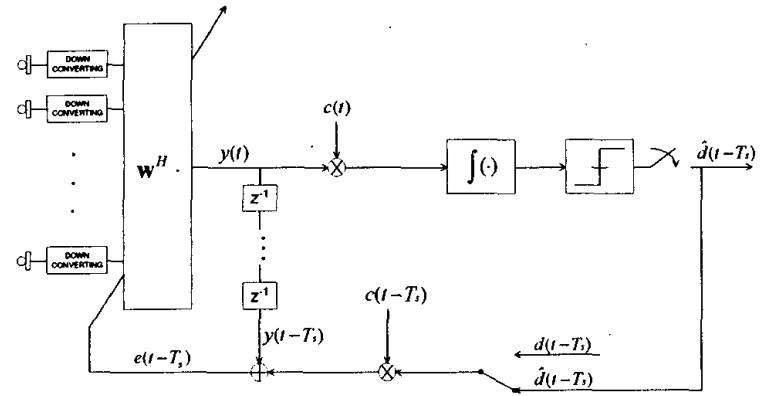

Figure 1: Scheme of the decision-directed beamforming method for DS-CDMA systems.

based on the minimization of the mean squared error (MSE), is given by [10]:

$$
\mathbf{w}_{\text {opt }}=\mathbf{R}_{x}^{-1} \mathbf{p}
$$

where $\mathbf{R}_{x}=E\left\{\mathbf{x}(t) \mathbf{x}^{H}(t)\right\}$ and $\mathbf{p}=E\left\{\mathbf{x}(t) d^{*}(t)\right\}$. Note that in a DS-CDMA context, the estimation of $\mathbf{R}_{x}$ and $\mathbf{p}$ is performed on a chip basis.

The main drawback of this method is the loss of spectral efficiency and channel bandwidth utilization due to the transmission of the training sequence. During the transmission of the data bits, a decision-directed approach can be used to continue adapting the beamformer. It basically exploits the finite alphabet restoral property to use the decided symbols as a training sequence [11].

The structure of this algorithm for a DS-CDMA system is depicted in Figure 1, where the decided symbols are spread again to be used as a reference at a chip level. It is important to note that this method requires PN synchronization.

\section{SELF-REFERENCE BEAMFORMER}

The self-reference beamformer is a blind cross-correlation algorithm for DS-CDMA systems that allows optimum beamforming in the sense of maximum SINR. It exploits the existence of temporal redundancy within the spread desired signal.

Assuming a spreading factor of $N_{c}$ chips, a block snapshot is defined by grouping columnwise the $N_{c}$ snapshots corresponding to one symbol, $\mathbf{X}=\left[\mathbf{x}_{1}, \mathbf{x}_{2}, \cdots, \mathbf{x}_{N_{c}}\right] \in \mathbb{C}^{\mathbf{Q x N}}$, being $\mathrm{Q}$ the number of antenna elements.

Each symbol can be seen, indeed, as a temporal repetition of a chip (with possible changes of sign due to the spreading signature). A first step in the exploitation of these temporal replicas is to polarize them, i.e., eliminate the changes of signs multiplying the signal by the synchronized spreading sequence of the desired user, $\mathbf{c} \in \mathbb{C}^{\mathbf{N}_{c} \times 1}$, to yield $\mathbf{X}^{p o l}=\mathbf{X} \cdot \operatorname{diag}(\mathbf{c})$, being $\operatorname{diag}(\mathbf{v})$ a diagonal matrix containing $v$ along its diagonal. Assuming that long PN sequences are used, i.e., the spreading codes change from symbol to symbol, then, in $\mathbf{X}^{\text {pol }}$ only the desired signal is fully correlated among the $N_{c}$ branches, whereas neither the interferences nor the noise are. This will be the key feature of the beamforming procedure (see Figure 2).

The polarized snapshots can be expressed as:

$$
\mathbf{x}_{k}^{\text {pol }}(t)=\sqrt{p_{d}} s_{d}(t) \mathbf{a}_{d}+\sum_{i=1}^{N_{I}} \sqrt{p_{i}} s_{i, k}(t) \mathbf{a}_{i}+\mathbf{n}_{k}(t)
$$

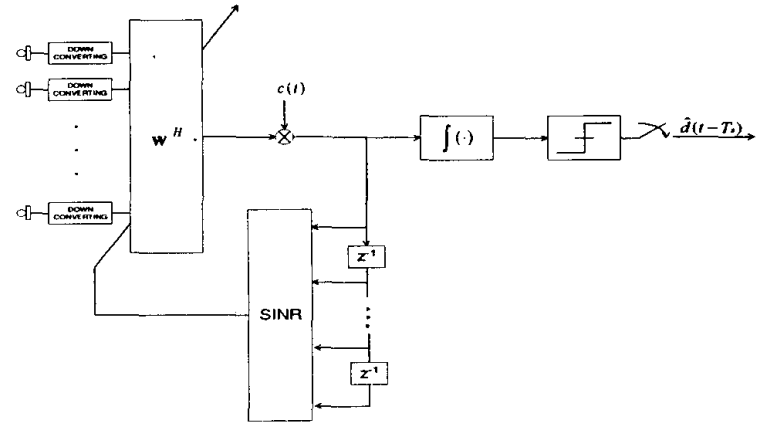

Figure 2: Scheme of the cross-correlation based beamforming method for DS-CDMA systems.

where $k$ denotes the branch $\left(1 \leq k \leq N_{c}\right), N_{I}$ is the number of interfering signals, $\mathbf{a}_{d}$ and $\mathbf{a}_{i}$ are the steering vectors (spatial signatures) of the desired and $i$ th interfering signal respectively, $s_{i, k}(t)$ is the corresponding direct sequence modulated $i$ th signal with power $p_{i}$ and $\mathbf{n}_{k}(t)$ is the additive white Gaussian noise vector in branch $k$.

The following equations are easily derived assuming uncorrelation among branches $E\left\{s_{i, k}(t) s_{j, l}(t)\right\}=\delta_{i j} \delta_{k l}$ (except for the desired user), which can be guaranteed due to the time-varying nature of the spreading codes:

$$
\begin{aligned}
& E\left\{\mathbf{x}_{l}^{p o l}(t) \mathbf{x}_{m}^{p o l, H}(t)\right\}=p_{d} \mathbf{a}_{d} \mathbf{a}_{d}^{H} \quad \text { for } \quad l \neq m \\
& E\left\{\mathbf{x}_{l}^{p o l}(t) \mathbf{x}_{l}^{p o l, H}(t)\right\}=p_{d} \mathbf{a}_{d} \mathbf{a}_{d}^{H}+\sum_{i=1}^{N_{I}} p_{i} \mathbf{a}_{i} \mathbf{a}_{i}^{H}+\sigma_{n}^{2} \mathbf{I}
\end{aligned}
$$

Therefore, the desired and signal covariance matrices can be efficiently obtained from the polarized snapshots of the $N_{c}$ diversity branches:

$$
\begin{aligned}
& \mathbf{R}_{d}=\frac{1}{N_{c}\left(N_{c}-1\right)} \sum_{l=1}^{N_{c}} \sum_{\substack{m=1 \\
m \neq l}}^{N_{c}} E\left\{\mathbf{x}_{l}^{p o l}(t) \mathbf{x}_{m}^{p o l, H}(t)\right\} \\
& \mathbf{R}_{\boldsymbol{x}}=\frac{1}{N_{c}} \sum_{k=1}^{N_{c}} E\left\{\mathbf{x}_{k}^{\text {pol }}(t) \mathbf{x}_{k}^{p o l, H}(t)\right\}
\end{aligned}
$$

which can be compactly expressed using the block snapshot previously defined:

$$
\begin{aligned}
\mathbf{R}_{\boldsymbol{d}} & =\frac{1}{N_{c}\left(N_{c}-1\right)} E\left\{\mathbf{X}^{p o l}(t) \tilde{\mathbf{I}} \mathbf{X}^{p o l, H}(t)\right\} \\
\mathbf{R}_{x} & =\frac{1}{N_{c}} E\left\{\mathbf{X}^{p o l}(t) \mathbf{X}^{p o l, H}(t)\right\}
\end{aligned}
$$

where the matrix $\tilde{\mathbf{I}} \in \mathbb{C}^{\mathrm{N}_{c} \times \mathbf{N}_{c}}$ is defined as $\tilde{\mathbf{I}}=\mathbf{1}-\mathbf{I}$, being $\mathbf{1}$ the all-one matrix and $\mathbf{I}$ the identity matrix. It is important to remark that matrices (7)-(8), derived from a new viewpoint of crosscorrelation properties at a chip level, are equivalent to those obtained from a complete different perspective of matched filtering by Suard et al. in [12], where a rank-one approximation was made.

The PN-based beamforming method will be based on the maximization of the following Rayleigh quotient:

$$
\operatorname{SINR}=\frac{\mathbf{w}^{H} \mathbf{R}_{d} \mathbf{w}}{\mathbf{w}^{H} \mathbf{R}_{i n} \mathbf{w}} \equiv \frac{\mathbf{w}^{H} \mathbf{R}_{d} \mathbf{w}}{\mathbf{w}^{H} \mathbf{R}_{x} \mathbf{w}}
$$




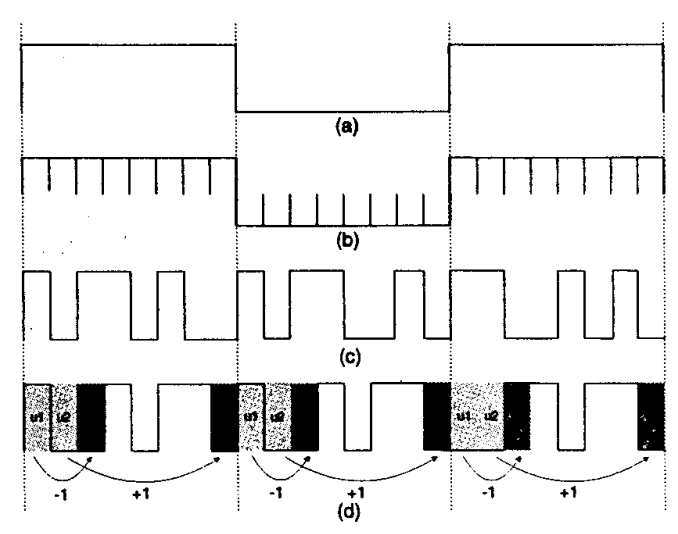

Figure 3: Example of Time Diversity in DS-CDMA: (a) original bit sequence, (b) bit sequence interpreted as a diversity repetition code, (c) spread bit sequence using a standard PN, and (d) spread bit sequence using a fixed redundancy structure (redundant PN).

which is given by the eigenvector corresponding to the maximum eigenvalue:

$$
\mathbf{R}_{d} \mathbf{w}=\lambda_{\max } \mathbf{R}_{x} \mathbf{w}
$$

The main drawback of this self-reference method (and of the TRB one as well) is the need of PN synchronization. For situations in which the spatial filtering is needed before complete synchronization is achieved, a modified algorithm with a more relaxed synchronization requirement has to be used.

The method proposed in [15] works in a similar way to the approach described herein, but introduces explicitly a redundancy structure. Although the original spreading code is time-varying in nature, the structure introduced remains fixed along the symbols (see Figure 3). Thus, only symbol synchronization is required and the method can work without global PN synchronization.

The method works on a block basis and uses a redundancy matrix different for each user (which simply repeats some chips with a possible change of sign) $\mathbf{G}^{T} \in \mathbb{C}^{\mathrm{MxN}}$ to convert a regular PN. frame $\mathbf{p n}_{q} \in \mathbb{C}^{\mathrm{N \times 1}}$ into a redundant (RPN) one $\mathbf{r p n}_{q} \in \mathbb{C}^{\mathrm{Mx} 1}$.

$$
\mathbf{r p n}_{q}=\mathbf{G}^{T} \mathbf{p} \mathbf{n}_{q}
$$

The receiver will make use of a parity check matrix, $\mathbf{H} \in \mathbb{C}^{\mathrm{MxR}}$ to define an error:

$$
\varepsilon_{q}=\mathbf{H}^{T} \mathbf{y}_{q}=\left(\mathbf{H}_{u}^{T}-\mathbf{H}_{v}^{T}\right) \mathbf{y}_{q}=\mathbf{u}_{q}-\mathbf{v}_{q}
$$

where $\mathbf{y}_{q} \in \mathbb{C}^{\mathrm{Mx1}}$ contains the desired modulated RPN signal plus noise and interferences (defined on a block basis). The matrices $\mathbf{H}_{u}$ and $\mathbf{H}_{v}$ can be seen as matrices that extract two different sets of diversity elements, $\mathbf{u}$ and $\mathbf{v}$, from the redundant vector $\mathbf{y}$ Note that the previously described method (PN-based beamforming), which uses the implicit redundancy of the spreading codes, is a particular case of this general scheme (RPN-based beamforming) with specific matrices $\mathbf{G}$ and $\mathbf{H}$.

As before, the key feature of the algorithm is the possibility of estimating the following matrices:

$$
\begin{aligned}
& \mathbf{R}_{d}=E\left\{\mathbf{X}_{q} \mathbf{H}_{u} \mathbf{H}_{v}^{H} \mathbf{X}_{q}^{H}\right\}+E\left\{\mathbf{X}_{q} \mathbf{H}_{v} \mathbf{H}_{u}^{H} \mathbf{X}_{q}^{H}\right\} \\
& \mathbf{R}_{x}=E\left\{\mathbf{X}_{q} \mathbf{H}_{u} \mathbf{H}_{u}^{H} \mathbf{X}_{q}^{H}\right\}+E\left\{\mathbf{X}_{q} \mathbf{H}_{v} \mathbf{H}_{v}^{H} \mathbf{X}_{q}^{H}\right\}
\end{aligned}
$$

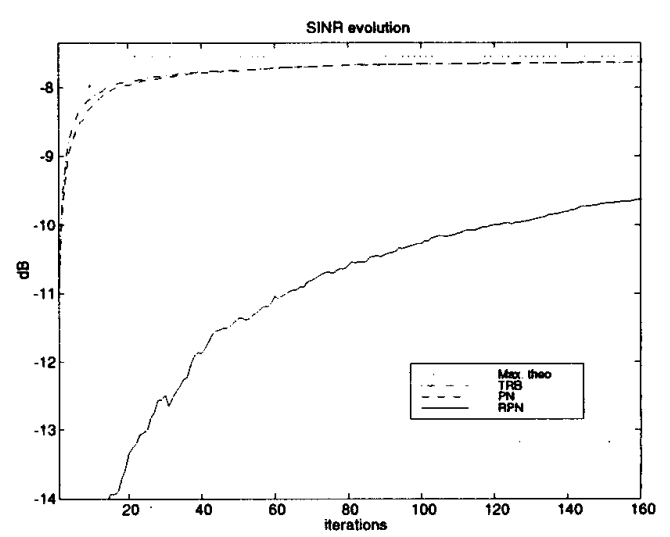

Figure 4: Ensemble average of the SINR evolution during 160 symbols for an array of 7 elements with the following scenario: desired signal with DOA of $15^{\circ}\left(E_{b} / N_{0}=5 \mathrm{~dB}\right)$ and 4 interfering signals impinging from angles of $0^{\circ},-30^{\circ}, 50^{\circ}$ and $80^{\circ}$ yielding a $I_{0} / N_{0}$ per sensor of $25.6 \mathrm{~dB}$. Gold sequences of length $N_{c}=1023$ and $\mathrm{SF}=31$ were used.

Note, however, that whereas $N_{c}\left(N_{c}-1\right) / 2$ different terms per symbol were used to estimate $\mathbf{R}_{d}$ in the PN-based algorithm, only $\mathrm{R}=\mathrm{M}-\mathrm{N}$ are used for the RPN-based method. This is an important fact for the convergence speed of the methods.

For both methods, the computationally expensive calculation of the generalized eigenvector can be efficiently implemented with a gradient-type adaptive algorithm minimizing the Lagrangian of the constrained minimization problem, yielding the following adaptation rule [16]:

$$
\mathbf{w}(n+1)=\mathbf{w}(n)-\boldsymbol{\mu}\left(\mathbf{R}_{d}-\lambda_{n} \mathbf{R}_{x}\right) \mathbf{w}(n)
$$

where $\mu$ is the step size parameter that controls both convergence and stability of the algorithm and $\lambda_{n}$ must be adaptively estimated as a quotient of powers [16]. Another possibility is the Power Iteration Method [17], which allows the calculation of the eigenvector corresponding to the eigenvalue with greatest magnitude:

$$
\mathbf{R}_{x}^{-1} \mathbf{R}_{d} \mathbf{W}=\lambda_{\max } \mathbf{W}
$$

where the inverse matrix $\mathbf{R}_{x}^{-1}$ can be efficiently computed on an adaptive fashion using the matrix inversion lemma.

\section{SIMULATIONS}

Monte Carlo simulations were performed with a uniform linear array (ULA) of 7 sensors in a hostile scenario to test the robustness of the beamforming algorithms. The desired signal had a direction of arrival (DOA) of $15^{\circ}$ and 4 interfering users impinged from angles of $-30^{\circ}, 5^{\circ}, 20^{\circ}$ and $50^{\circ}\left(E_{b} / N_{o}\right.$ of $30 \mathrm{~dB}, 20 \mathrm{~dB}, 40 \mathrm{~dB}$ and $20 \mathrm{~dB}$ ) respectively. Gold sequences of length $N_{c}=1023$ chips and and $\mathrm{SF}=31$ were utilized for both methods PN/RPN-based beamforming.

The ensemble SINR evolution of the three previously described methods (TRB+DD using $10 \%$ as training sequence, PN-based, RPN-based) during 160 symbols (updating the estimated matrices 


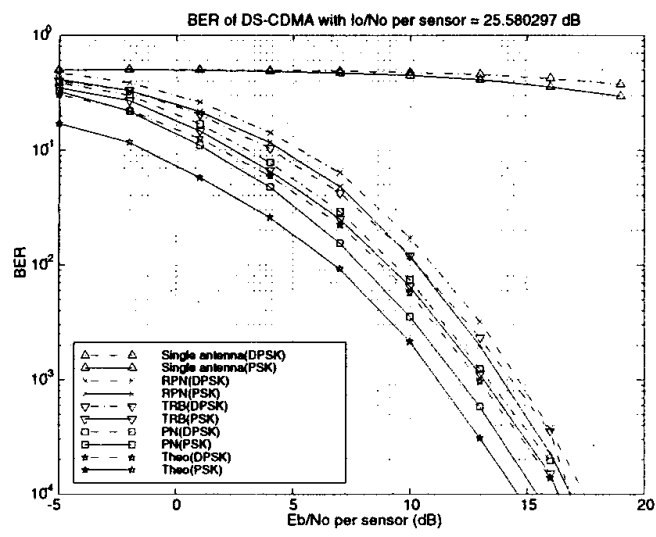

Figure 5: Uncoded BER of the different methods (TRB+DD using $10 \%$ as training sequence, PN-based, RPN-based) along with the theoretically maximum for BPSK and binary DPSK. The scenario was composed of a desired signal with DOA of $15^{\circ}$ and 4 interfering signals with DOA's of $0^{\circ},-30^{\circ}, 50^{\circ}$ and $80^{\circ}\left(E_{b} / N_{o}\right.$ of $30 \mathrm{~dB}$, $20 \mathrm{~dB}, 40 \mathrm{~dB}$ and $20 \mathrm{~dB}$ ) respectively. $N_{c}=1023$ and $\mathrm{SF}=31$.

at each new received symbol) is depicted in Figure 4 for $E_{b} / N_{0}=$ $5 \mathrm{~dB}$. It can be seen how the convergence time of the PN-based method is almost identical to that of the TRB. Regarding the RPNbased method, it clearly shows a longer convergence time due to the small number of cross-correlation terms used $(R=2)$ compared to the 465 terms in the PN case. It is important to note that the given output SINR's were computed before the despreading stage (at chip level), which theoretically would give an increase of $10 \log _{10} \mathrm{SF} \simeq 15 \mathrm{~dB}$.

In Figure 5, the performance in terms of uncoded BER of all methods for BPSK and binary DPSK is given. It can be seen how the PN-based method outperforms the TRB and is about $1 \mathrm{~dB}$ worse than the theoretical minimum BER. The RPN-based method is inferior to the other two methods. Therefore, the proposed method clearly outperforms the RPN-based one and the TRB (using 10\% of the chips as a training sequence).

\section{CONCLUSIONS}

An adaptive self-reference beamforming algorithm particularly intended for DS-CDMA systems has been derived from a novel perspective of chip cross-correlation properties. The method is based on the implicit temporal redundancy structure of spreading codes (PN-based). It has been compared to the classical TRB and to a previously presented method based on a temporal redundancy explicitly introduced (RPN-based).

Simulation results have shown the effectivity of the proposed scheme, outperforming the TRB in terms of BER without the need of side information (training sequence), which implies a better spectral efficiency and channel bandwidth utilization.

The method is robust to antenna element calibration errors and to multipath environment because it does not make any assumption regarding the structure of the steering vectors.

\section{REFERENCES}

[1] T. IS-95A, Mobile Station-Base Station Compatibility Standard for Dual Mode Spread Spectrum Cellular System. 1994.

[2] V. K. Garg, L. Smolik, and J. E. Wilkes, Applications of Code-Division Multiple Access (CDMA) in Wireless/Personal Communications. Upper Saddle River, NJ: Prentice Hall, 1996.

[3] S. Moshavi, "Multi-user detection for DS-CDMA communications," IEEE Comm. Magazine: Topics in Personal Communications, pp. 124-136, Oct 1996.

[4] S. Verdú, "Minimum probability of error for asynchronous gaussian multiple-access channels," IEEE Trans. on Information Theory, vol. IT-32, pp. 85-96, January 1986.

[5] R. Lupas and S. Verdú, "Linear multiuser detectors for synchronous code-division multiple access channel," IEEE Trans. on Information Theory, vol. IT-35, pp. 123-136, January 1989.

[6] R. Lupas and S. Verdú, "Near-far resistance of multiuser detectors in asynchronous channel," IEEE Trans. on Communications, vol. COM-38, pp. 496-508, April 1990.

[7] X. Xie, C. K. Rushforth, and R. T. Short, "Multiuser signal detection using sequential decoding," IEEE Trans. on Communications, vol. 38, pp. 578-583, May 1990.

[8] S. Moshavi, E. G. Kanterakis, and D. L. Schilling, "Multistage linear receivers for DS-CDMA systems," Int. J. Wireless Info. Networks, vol. 3, Jan 1996.

[9] M. K. Varanasi and B. Aazhang, "Multistage detection in asynchronous code-division multiple-access communications," IEEE Trans. on Communications, vol. 38, pp. 509519, April 1990.

[10] R. A. Monzingo and T. W. Miller, Introduction to adaptive arrays. New York Wiley cop., 1980.

[11] A. L. Swindlehurst, S. Daas, and J. Yang, "Analysis of a decision directed beamformer," IEEE Trans. on Signal Processing, vol. 43, pp. 2920-2927, December 1995.

[12] B. Suard, A. F. Naguib, G. Xu, and A. Paulraj, "Performance of CDMA mobile communication systems using antenna arrays," in Proceeding of the IEEE ICASSP-93, (Minneapolis), pp. IV153-IV156, April 1993.

[13] J. Choi, "Beamforming for the multiuser detection with decorrelator in synchronous CDMA systems: Approaches and performance analysis," Signal Processing, vol. 60, pp. 195-212, July 1997.

[14] O. Muñoz and J. Fernández-Rubio, "Blind multiuser combining at the base station for asynchronous CDMA systems," in Proc. IEEE ICASSP '96, (Atlanta, USA), pp. 2710-2713, May 1996.

[15] D. Pérez-Palomar and M. A. Lagunas, "Blind beamforming for DS-CDMA systems," Proc. Fifth Bayona Workshop on Emerging Technologies in Telecomm., pp. 83-87, Sept 1999.

[16] M. Nájar, X. Mestre, and M. A. Lagunas, "Two-stage code reference beamformer for the reception of frequency hopping modulated signals," Accepted at Eurasip Signal Processing.

[17] G. H. Golub and C. F. V. Loan, Matrix Computations. The Johns Hopkins University Press, 1996. 